\title{
Performance analysis of IEEE 802.11ac based WLAN in wireless communication systems
}

\author{
A. Z. Yonis \\ College of Electronics Engineering, Ninevah University, Mosul, Iraq
}

\begin{tabular}{|c|c|}
\hline Article Info & ABSTRACT \\
\hline Article history: & IEEE $802.11 \mathrm{ac}$ based wireless local area network (WLAN) is emerging WiFi \\
\hline Received Jan 27, 2018 & $\begin{array}{l}\text { standard at } 5 \mathrm{GHz} \text {, it is new gigabit-per-second standard providing premium } \\
\text { services. IEEE } 802.11 \mathrm{ac} \text { accomplishes its crude speed increment by pushing }\end{array}$ \\
\hline Revised May 28, 2018 & on three distinct measurements firstly is more channel holding, expanded \\
\hline Accepted Jun 15, 2018 & $\begin{array}{l}\text { from a maximum of } 80 \mathrm{MHz} \text { up to } 160 \mathrm{MHz} \text { modes. Secondly, the denser } \\
\text { modulation, now using } 256-\mathrm{QAM} \text {, it has the ability to increase the data rates }\end{array}$ \\
\hline Keywords: & $\begin{array}{l}\text { up to } 7 \mathrm{Gbps} \text { using an } 8 \times 8 \text { multiple input multiple output (MIMO). Finally, it } \\
\text { provides high resolution for both narrow and medium bandwidth channels. }\end{array}$ \\
\hline $\begin{array}{l}\text { IEEE } 802.11 \\
\text { Modulation }\end{array}$ & $\begin{array}{l}\text { This work presents a study to improve the performance of IEEE 802.11ac } \\
\text { based WLAN system. }\end{array}$ \\
\hline
\end{tabular}

Copyright () 2019 Institute of Advanced Engineering and Science. All rights reserved.

Corresponding Author:

A. Z. Yonis,

College of Electronics Engineering,

Ninevah University,

Mosul, Iraq.

Email: aws_zuher@yahoo.com

\section{INTRODUCTION}

Recently, wireless communications have fundamentally developed because of the trend setting innovation of cell phones, convenient gadgets and the fast development of Internet of Things (IOT) [1], astute transportation frameworks and person to person communication. IEEE 802.11 is a well-known standard that widely used to control the physical layer determinations for executing wireless local area network (WLAN) computer for various frequency bands such as $900 \mathrm{MHz}$ and 2.4, 3.6, 5, and $60 \mathrm{GHz}$ [2].

At 1997 the basics of IEEE 802.11 was discharged and then the results were revised. This system with its corrections are focusing on the items of wireless systems such as Wi-Fi. On the other hand, every modification is revoked when it is established in the last version of the system. The companies resort to market to get the corrections and updates in order to improve the capabilities of products. These revised systems will become new standards. Wireless communications heading towards high bandwidth of data transmission for multi-users at the same time. IEEE 802.11 standards evolution is shown in Figure 1.

This paper introduces the history of IEEE 802.11 standard in section 2 . While section 3 presents how IEEE 802.11 developped and improved to IEEE 802.11ad.The simulation results with detailed discussions are presented in section 4. Finally, the important conclusions and some suggestions for further improvement of the proposed work are discussed in section 5. 


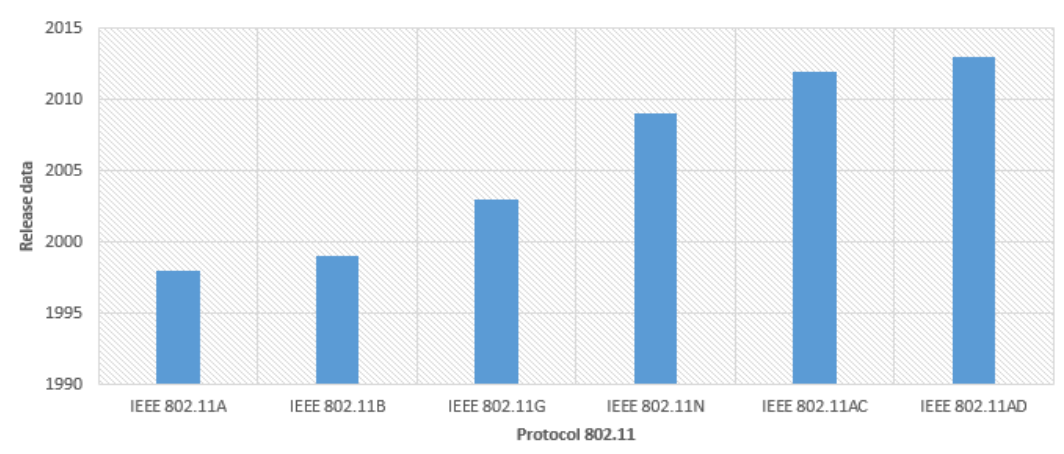

Figure 1. IEEE 802.11 development from IEEE 802.11A to IEEE 802.11ad

\section{HISTORY OF IEEE 802.11 STANDARD}

IEEE 802.11a utilizes similar information connect layer convention and casing group to the first one even the used air interface is OFDM. This IEEE standard is working on $5 \mathrm{GHz}$ band also the peak data rate reaches to $54 \mathrm{Mbps}$. On the other hand, the throuhput is around $20 \mathrm{Mbps}$ and this value is obtained according to the code of error correction distinctive correspondence frameworks such as GSM, LTE and WLAN which give remote web access to assortment of uses.

The utilization of mobile as an instrument to access the services of internet daily turns out to be more pressing and fruitful advancement of mobile wireless technology builds the capacity to make high throughput and minimal effort systems for the client in-movement. Giving intelligent transportation frameworks stable wireless internet association has turned into an appealing examination field for a long time. Among the numerous innovations suggested for frameworkswith intelligent transmission, wireless communications and vehicle to individual correspondence, means to build drivers and travelers comfort, street security and transport efficiency and give pervasive wireless network to the internet [4]. While IEEE 802.11b has a most extreme crude information rate of $11 \mathrm{Mbps}$, and furthermore utilizes similar media access. IEEE $802.11 \mathrm{~b}$ items showed up on 2000, Wireless communications are ending up progressively imperative for their flexibility, portability and speed. It is the situation of microwave and laser based innovations, e.g. Wi-Fi and free space optics, individually. The significance and usage of Wi-Fi have been developing for supplementing conventional wired systems. Wi-Fi has had an expanding nearness in the individual home, shaping a remote individual territory arrange, enabling individual gadgets to impart. Pointto-point and point-to-multipoint arrangements are utilized both inside and outside, requiring specific directional and omnidirectional radio wires [5].

Confirmed the IEEE 802.11g in 2003 it works (like IEEE 802.11b), in the $2.4 \mathrm{GHz}$ band, likewise utilizes the same OFDM based transmission. IEEE $802.11 \mathrm{~g}$ is the third adjustment standard for remote LANs. This standard is working in $2.4 \mathrm{GHz}$ band and has raw data rate of $54 \mathrm{Mbps}$. The throughput of this IEEE $802.11 \mathrm{~g}$ reachs $31.4 \mathrm{Mbps}$ if the used multiple access depends the scheme of avoiding collisionin transmission; this troughput is for 1500 bytes with wireless rate of $54 \mathrm{Mbps}$. In fact, this throughput can not be achieved practically due to the implementation of access points, so throughput of packets with size less than 1500 bytes will have throughput around $3 \mathrm{Mbps}$ if the used rate is $54 \mathrm{Mbps}$ and the packets have 64 bytes [6].

IEEE 802.11 based WLANs are generally utilized in view of its adaptability; Higher throughput can be achieved by upgrading the execution medium access control layer all things considered medium access control layer (MAC) outline total component can be utilized. This standard has two types of casing total: MAC protocol data unit aggregation and the other is MAC service data unit aggregation [7].

IEEE $802.11 \mathrm{n}$ is a change that enhances the past IEEE 802.11 standards by including MIMO antennas i.e. multiple input antennas and multiple output antennas. On the other hand, IEEE 802.11n is working on two bands: $2.4 \mathrm{GHz}$ and $5 \mathrm{GHz}$. The peak data rate of this standard may reach $600 \mathrm{Mbps}$ intead of $54 \mathrm{Mbps}$ as confirmed bt IEEE and distributed in 2009. Finally, in 2013 IEEE 802.11 is revised to IEEE 802.11 ac. It constructed in light of the $802.11 \mathrm{n}$ standard. also, standard is distributed in 2013, which expands on IEEE $802.11 \mathrm{n}$ and it guarantees that throughput has high level, it utilizes high transfer speed and high quantities of MIMO, and high requests of modulations [8]. 


\section{IEEE 802.11AD STANDARD FEATURES}

The physical layer of IEEE 802.11 networks is characterized efficienty through IEEE 802.11ad. This standard is developed so that it can work on millimeter wave with range of $60 \mathrm{GHz}$. The characteristics of this band is fully unique and this band of frequency differs from $2.4 \mathrm{GHz}$ and $5 \mathrm{GHz}$ in the conditions of Wi-Fi network systems. All the items that excuting this standard (IEEE 802.11ad) are carried under the name of WiGig brand. IEEE 802.11ad basics are depending the protocol of hybrid MAC (medium access control) which ensures avoiding the collisions and non-cnflict based channel instruments. It additionally utilizes directional receiving ways to work on $60 \mathrm{GHz}$ with minimum losses in path.So, IEEE 802.11ad is realy different and contrasts if it is compared to other standards such as IEEE $802.11(\mathrm{~b} / \mathrm{g} / \mathrm{n})$ because this standard needs new strategies to investigate its execution [9]. The genuine test for the IEEE 802.11ax standards improvement is to outperform from perspective of bandwidth in an initial step and after that as in general execution the Ethernet standard [10], [11].

\section{SIMULATION AND RESULTS}

The simulation supports the IEEE 802.11a, IEEE 802.11n and IEEE 802.11ac standards with an extensive variety of settings. A block diagram showing the fundamental circle of the simulation is shown in Figure 2. The following subsections clarify each block in more subtle details.

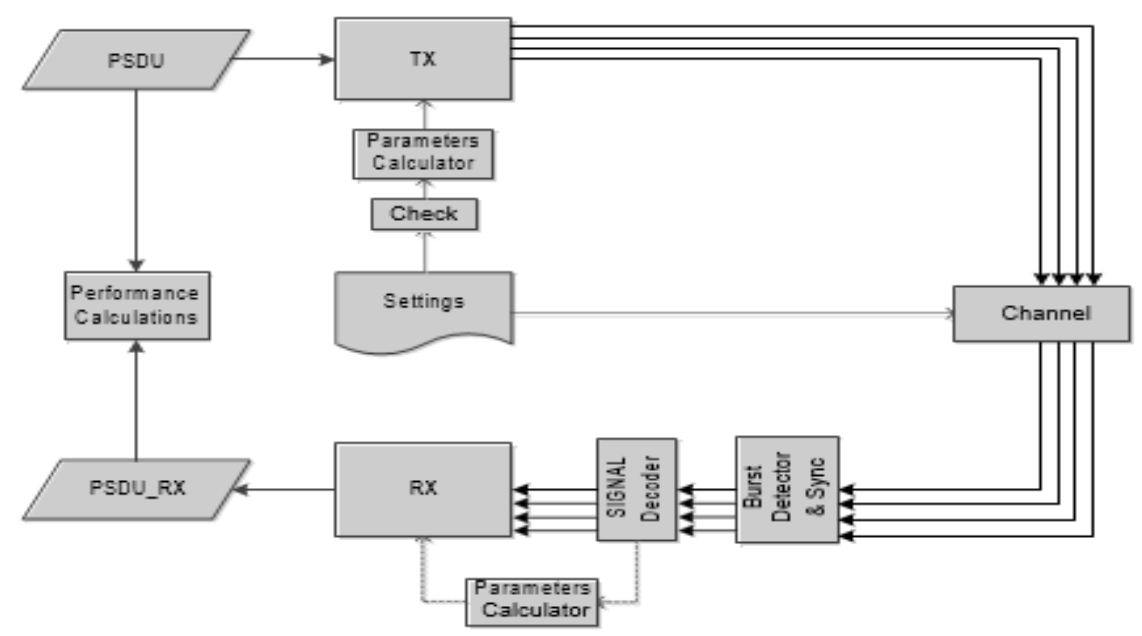

Figure 2. Block diagram of the simulation 7

As shows in Figure 3(a), we used type of modulation called BPSK with MSC (0) and code rate; With code rate $1 / 2$ and we noticed that the maximum value of bit error rate is $9.98 \mathrm{e}-06 \mathrm{~dB}$ when single noise ratio (SNR) is $\mathrm{dB}$ and in theoretical when SNR is $10 \mathrm{~dB}$ the value of BER is 3.872e-06.

As shows in Figure 3(b), we used type of modulation called with QPSK 3/4 MSC (2) and code rate $3 / 4$ and we noticed that the maximum value of bit error rate is $9.98 \mathrm{e}-5 \mathrm{~dB}$ when single noise ratio (SNR) is $10 \mathrm{~dB}$ and in theoretical when SNR is $15 \mathrm{~dB}$ the value of BER is 9.361e-09.

As shows in Figure 3(c), we used type of modulation called with 16 QAM 1/2 MSC (3) and code rate $1 / 2$ and we noticed that the maximum value of bit error rate is $1.999 \mathrm{e}-05$ when single noise ratio (SNR) is $20 \mathrm{~dB}$ and in theoretical when SNR is $20 \mathrm{~dB}$ the value of BER is $1.999 \mathrm{e}-05$.

Figure 4(a), we used type of modulation called with 16 QAM 3/4 MSC (4) and code rate 3/4 and we noticed that the maximum value of bit error rate is 0.0043 when single noise ratio (SNR) is $15 \mathrm{~dB}$ and in theoretical when SNR is $20 \mathrm{~dB}$ the value of BER is $2.904 \mathrm{e}-06$. Figure 4(b), we used type of modulation called with 64 QAM 2/3 MSC (5) and code rate 2/3 and we noticed that the maximum value of bit error rate is $4.999 \mathrm{e}-05$ when single noise ratio (SNR) is $25 \mathrm{~dB}$ and in theoretical when SNR is $25 \mathrm{~dB}$ the value of BER is 4.999e-05 While Figure 4(c), we used type of modulation called with 64 QAM 3/4 MSC (6) and code rate $3 / 4$ and we noticed that the maximum value of bit error rate is $2 \mathrm{e}-05$ when single noise ratio ( $\mathrm{SNR}$ ) is $25 \mathrm{~dB}$ and in theoretical when SNR is $25 \mathrm{~dB}$ the value of BER 2e-05. Figure 5(a), we used type of modulation called with 64 QAM 5/6 MSC (7) and code rate 5/6 and we noticed that the maximum value of bit error rate is $3.999 \mathrm{e}-05$ when single noise ratio (SNR) is $25 \mathrm{~dB}$ and in theoretical when SNR is $25 \mathrm{~dB}$ the value of BER is $3.999 \mathrm{e}-05$.

Performance Analysis of IEEE 802.11ac based WLAN in Wireless Communication Systems (A.Z. Yonis) 


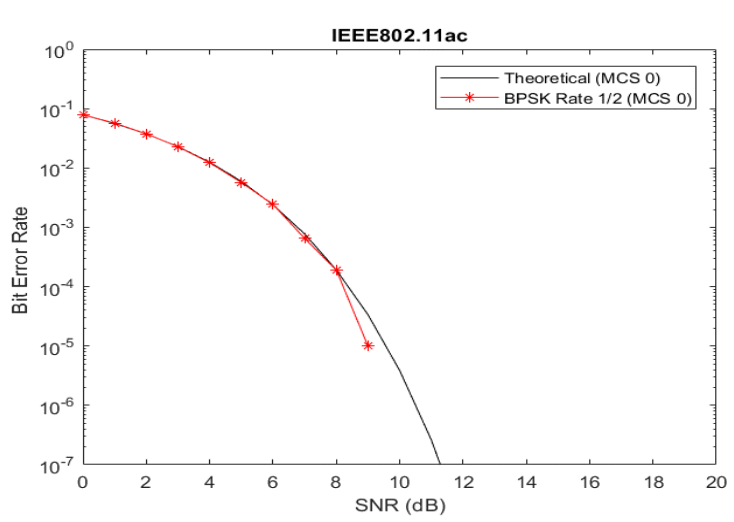

Figure 3(a). Simulation of IEE 802.11 ac with BPSK $1 / 2$

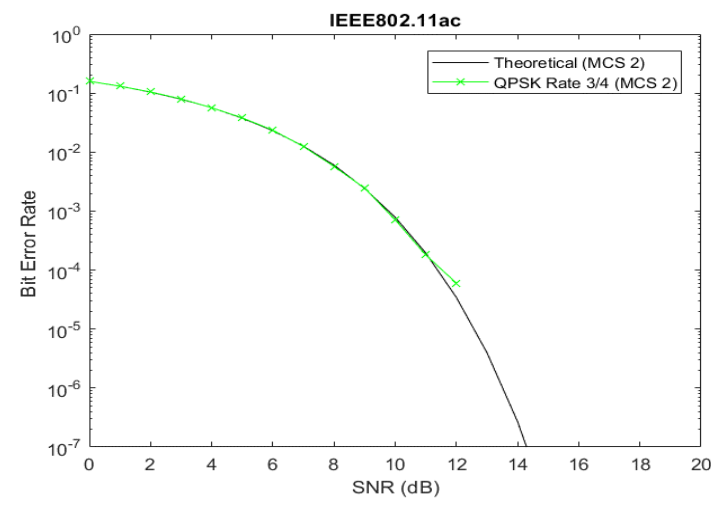

Figure 3(b). Simulation of IEEE 802.11ac with QPSK $3 / 4$

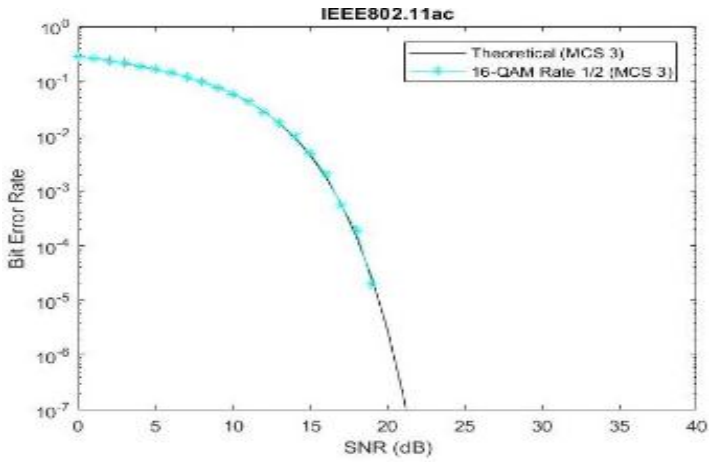

Figure 3(c). Simulation of IEEE802.11 ac with 16 QAM 1/2

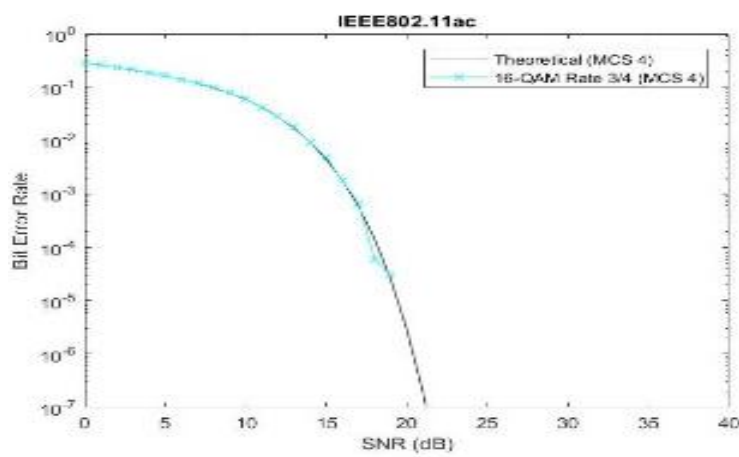

Figure 4(a). Simulation of IEEE 802.11 ac with 16QAM 3/4

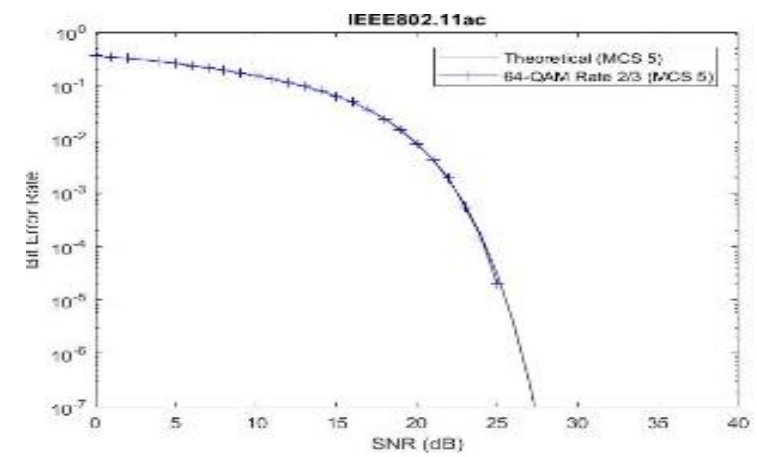

Figure 4(b). Simulation of IEEE 802.11ac with 64 QAM 2/3

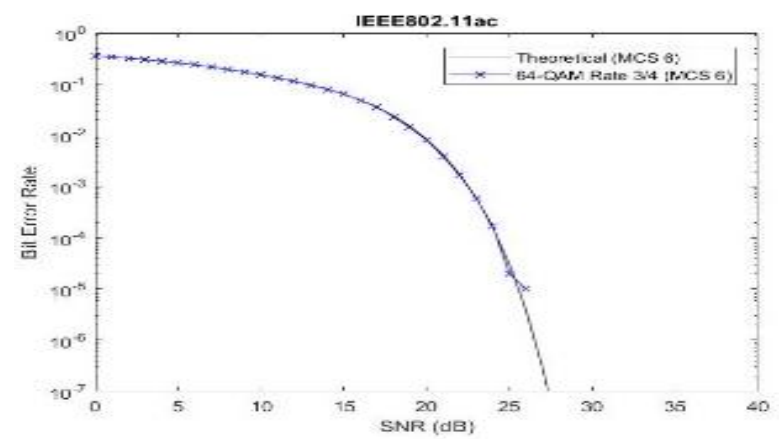

Figure 4(c). Simulation of IEEE 802.11ac with 64 QAM 3/4 


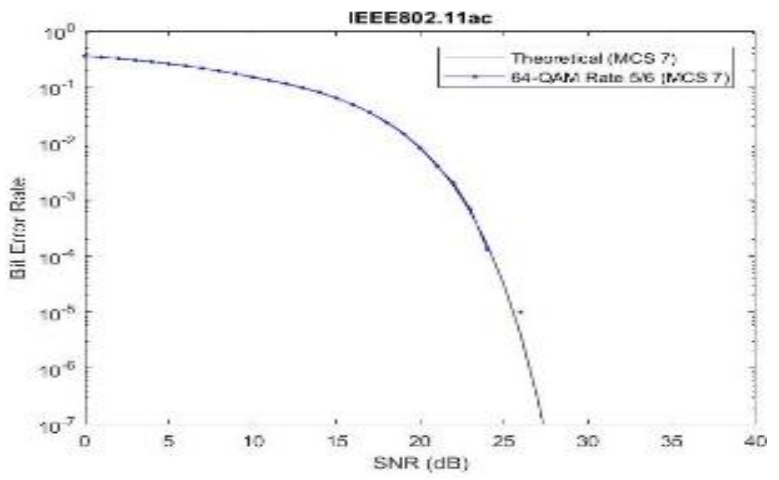

Figure 5(a). Simulation of IEEE 802.11ac with 64 QAM 5/6

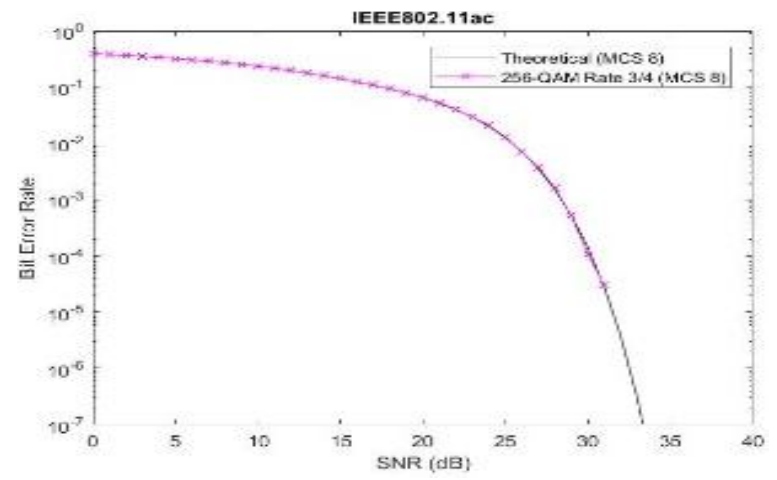

Figure 5(b). Simulation of IEEE 802.11ac with 256QAM

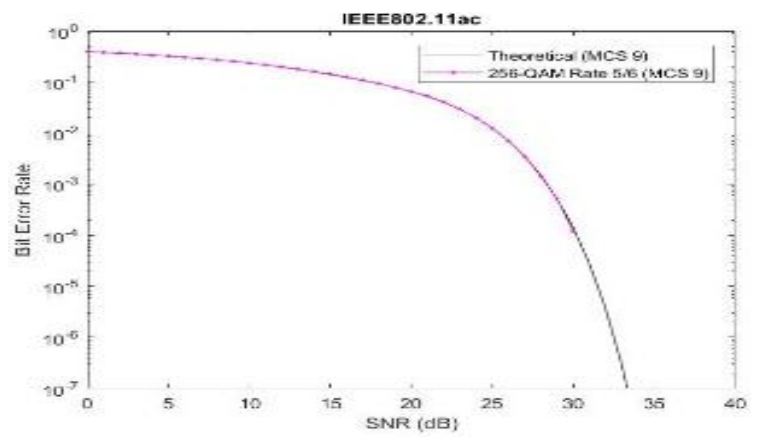

Figure 5(c). Simulation of IEEE 802.11ac with 256 QAM 5/6

Figure 5(b), we used type of modulation called with 256 QAM 3/4 MSC (8) and code rate 3/4 and we noticed that the maximum value of bit error rate is $4.4996 \mathrm{e}-05$ when single noise ratio (SNR) is $30 \mathrm{~dB}$ and in theoretical when SNR is $35 \mathrm{~dB}$ the value of BER $2.495 \mathrm{e}-10$ while Figure 5(c), we used type of modulation called with 256 QAM 5/6 MSC (9) and code rate 5/6 and we noticed that the maximum value of bit error rate is 0.00013 when single noise ratio (SNR) is $30 \mathrm{~dB}$ and in theoretical when SNR is $35 \mathrm{~dB}$ the value of BER 2.495e-10. As a result, Figure 6 summarizes the performance of the system with different modulation techniques. So it is preferable to use the BPSK in transmitting the data on the AWGN channel.

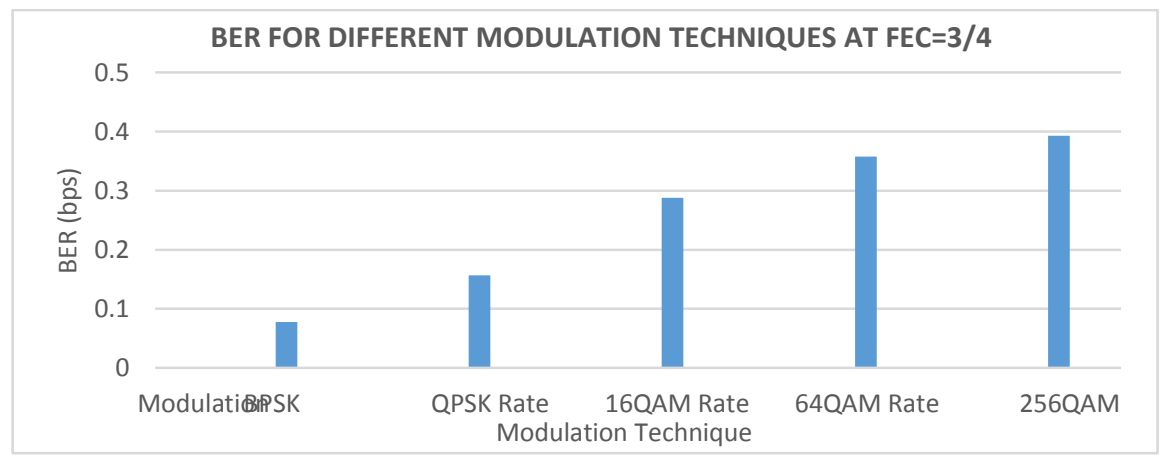

Figure 6. BER values for different modulation techniques

\section{CONCLUSIONS}

This paper presents an adequate analysis and results of the IEEE 802.11ac standard. It also shows its capability to efficiently perform with the modern communication systems with acceptable performance. IEEE 802.11 standards give portability support to voice correspondence, wireless information systems can be partitioned into a few sorts relying upon their zone of scope. Through range changes and speedier remote

Performance Analysis of IEEE 802.11ac based WLAN in Wireless Communication Systems (A.Z. Yonis) 
transmissions, IEEE 802.11ac, ad and 802.11ax promotion are enhancing the execution of superior quality TV and computerized video streams in the home and propelled applications in big business systems. They enhance generally client encounter where and at whatever point individuals are associated. All the applied improvements are designed to increase the speed of data transportation. On the other side, the frequency will be different because IEEE 802.11 ad uses unlicensed $60 \mathrm{GHz}$ band and shorter range transmissions. The research paper summarizes the execution of the framework with various modulation methods and it is desirable over utilize the BPSK adjustment method for transmission of the data information in the AWGN channel.

\section{ACKNOWLEDGMENT}

This research study is achieved in the department of Communication Engineering/College of Electronics Engineering at Ninevah University, Mosul, Iraq.

\section{REFERENCES}

[1] E. Y. Adesta, D. Agusman,and A. Avicenna, "Internet of Things (IoT) in Agriculture Industries," Indonesian Journal of Electrical Engineering and Informatics (IJEEI), Vol. 5, No. 4, 2017, pp. 376-382.

[2] M. Elkhodr, Q. F. Hassan and S. Shahrestani, "Networks of the Future: Architectures, Technologies, and Implementations," Book CRC, 2017.

[3] Y. Park and F. Adachi, "Enhanced Radio Access Technologies for Next Generation Mobile Communication," Book springer, pp.20. 2007.

[4] J. Jansons and A. Barancevs, "Using wireless networking for vehicular environment: IEEE 802.11a standard performance," 2nd IEEE International Conference on Digital Information Processing and Communications (ICDIPC), 2012, pp.5-9.

[5] J. A. Carvalho; H. Veiga; N. Marques; C.F.Pacheco, and A.D.Reis, "A Contribution to Laboratory Performance Measurements of IEEE 802.11 B,G Wep point-to-point Links," International Conference on Wireless Information Networks and Systems (WINSYS), 2010, pp.1-4.

[6] J. Jangeun, P. Pushkin, and S. Mihail, "Theoretical Maximum Throughput of IEEE 802.11 and its Applications," Second IEEE International Symposium on Network Computing and Applications, 2003, pp. 249-256.

[7] M. S. Kowsar and S. Biswas, "Performance improvement of IEEE 802.11n WLANs via frame aggregation in NS3," IEEE international conference on electrical, computer and communication engineering (ECCE), 2017, pp. 1-6.

[8] N. Al-Ghazu, "A Study of the Next WLAN Standard IEEE 802.11ac Physical Layer," M. S. Thesis Stockholm, Sweden, 2013.

[9] K. Chandra, R. V. Prasad, and I. Niemegeers, "Performance Analysis of IEEE 802.11ad MAC Protocol," IEEE communications letters, vol. 21, no. 7, 2017, pp. 1513-1516.

[10] D. Deng, K. Chen and R. Cheng, "IEEE 802.11ax: Next Generation Wireless Local Area Networks," $10^{\text {th }}$ International Conference on Heterogeneous Networking for Quality,Reliability,Security and Robustness, Rhodes, 2014, pp.77-82.

[11] M. P. Putra, D. Perdana and R. M. Negara, "Performance Analysis of Data Traffic Offload Scheme on Long Term Evolution and IEEE 802.11AH," TELKOMNIKA (Telecommunication Computing Electronics and Control), vol. 15, no. 4,2017 , pp. $1659-1665$. 